\title{
VARIATIONAL CALCULATION FOR TRIANGULAR QUANTUM WELLS USING MODIFIED TRIAL WAVE FUNCTION*
}

\author{
B. PöDöR \\ Research Institute for Technical Physics of the Hungarian Academy of Sciences \\ P.O. Box 76, 1325 Budapest, Hungary
}

In calculations involving triangular quantum wells the Fang-Howard variational wave function is widely used because of its simplicity, although it can lead to substantial errors. This can also occur in transport calculations, where the scattering rate, being proportional to the integral of the fourth power of the wave function, depends strongly on the shape of the wave function. A new set of one-parameter wave functions is introduced and the relaxation time is evaluated for short range scatterers. The improved wave function leads up to about 22 per cent increase in the scattering rate, and to a comparable decrease in the relaxation time.

PACS numbers: 72.20.Dp, 73.20.Dx

\section{Introduction}

The triangular potential approximation is widely used to describe energy bands in inversion layers and at single heterojunctions. While it allows the exact analytical solution of the Schrödinger equation in terms of Airy functions [1], in practical calculations, e.g. in those involving transport effects, simple approximate analytical wave functions are much more preferred and widespread. The simplest of these, originally proposed by Fang and Howard [2] for inversion layer calculations (for the relevant algebra cf. [3-6]),

$$
\begin{array}{ll}
\psi_{\mathrm{FH}}(z)=0 & \text { for } z \leq 0 \\
\psi_{\mathrm{FH}}(z)=\left(\frac{b^{3}}{2}\right)^{1 / 2} z \exp (-b z / 2) & \text { for } z>0
\end{array}
$$

contains only one variational parameter, $b$, which is determined by minimizing the total energy. Because of the simplicity of the wave function, it is easy to obtain the expectation value of the energy, and analytical results could also be found for many problems in the field of transport phenomena.

\footnotetext{
*This work was supported from grant No. CP93:8252 from the EC Copernicus Project.
} 
However, in many cases of energy band calculations the use of the FangHoward trial function can lead to substantial errors (for a review see [5]). This should be even more the case for transport calculations, where the expressions for the scattering rate and the inverse relaxation time contain the integral of the fourth power of the wave function $[5,6]$.

In order to calculate more accurate energies, Takada and Uemura [7] introduced another one-parameter trial wave function

$$
\begin{array}{ll}
\psi_{\mathrm{TU}}(z)=0 & \text { for } z \leq 0, \\
\psi_{\mathrm{TU}}(z)=\left(\frac{3 b^{3}}{2}\right)^{1 / 2} z \exp \left[-(b z)^{3 / 2} / 2\right] & \text { for } z>0,
\end{array}
$$

which gave a much more accurate energy for the ground subband, i.e. only 0.39 per cent above the exact Airy function value, compared with the error of 5.91 per cent when using the Fang-Howard wave function. This improvement was ascribed to the correct asymptotic behaviour of the Takada-Uemura wave function for large $z$ values [7]. Of course, many-parameter functions can also be used but with a loss of the transparency of calculations (see e.g. $[8,9]$ ).

In this paper we explore new one-parameter wave functions and evaluate the scattering rate and relaxation time for the simple case of short range scattering potentials. We will show that the improved wave functions lead to a substantially greater scattering rate and to a comparable decrease in the relaxation time as compared to the values based on formulas usually applied in the literature.

\section{Variational calculation}

Given a triangular quantum well described by the potential

$$
V(z)=\infty \text { for } z \leq 0 \text { and } V(z)=e F z \text { for } z>0 \text {, }
$$

consider now the following trial (quasi-)one-parameter normalized variational wave function

$$
\psi_{n(z)}=\left(\frac{n b_{n}^{3}}{\Gamma(3 / n)}\right)^{1 / 2} z \exp \left[-\left(b_{n} z\right)^{n} / 2\right] \quad \text { for } z>0
$$

and 0 elsewhere. Here $b_{n}$ is the variational parameter, $n$ is a real positive number, and $\Gamma(x)$ is the gamma-function. The $\psi_{n}(z)$ trial function is simple ad hoc generalization of the Fang-Iloward $(n=1)$ and the Takada-Uemura $(n=3 / 2)$ wave functions.

Using the trial function given in Eq. (4) the sum of the kinetic and potential energies in the potential given in Eq. (3) is easily calculated as

$$
\left\langle E_{n}\right\rangle=\left\langle T_{n}\right\rangle+\left\langle V_{n}\right\rangle=\frac{n(1+n)}{4} \frac{\Gamma(1+1 / n)}{\Gamma(3 / n)} b_{n}^{2} \frac{\hbar^{2}}{2 m^{*}}+\frac{\Gamma(4 / n)}{\Gamma(3 / n)} \frac{e f}{b_{n}} .
$$

Minimizing the total energy $\left\langle E_{n}\right\rangle$ with respect to $b_{n}$, we obtained

and

$$
b_{n \text { min }}^{3}=\frac{e F}{\hbar^{2} / 2 m^{*}} \frac{2}{n(1+n)} \frac{\Gamma(4 / n)}{\Gamma(1+1 / n)}
$$

$$
E_{n}=\frac{[\Gamma(4 / n)]^{2 / 3}}{\Gamma(3 / n)} \frac{3}{2}\left[\Gamma(1+1 / n) \frac{n(n+1)}{2}\right]^{1 / 3}\left(\frac{\hbar^{2}}{2 m^{*}}\right)^{1 / 3}(e F)^{2 / 3} .
$$


The energy minimum of $E_{n}\left(b_{n \min }\right)$ is now found for $n=1.749$, for which $E_{2 \min }=$ 2.33821 , which is only 0.0044 per cent greater than the exact (Airy function) value of 2.33810741 .

For the optimized trial function the spread of the wave function, $\langle\Delta z\rangle=$ $\left(\left\langle z^{2}\right\rangle-\langle z\rangle^{2}\right)^{1 / 2}$, agreed very closely with that of the exact (Airy) wave function, i.e. not the asymptotic behaviour, as surmised by Takada and Uemura [7], but the shape of the trial function itself which is relevant in this case.

\section{Relaxation time for short range potential scattering}

For short range scatterers, modelled with a $\delta$-function potential, the inverse relaxation time, being proportional to the integral of the fourth power of the wave function, is more sensitive to the exact shape of the wave function. As an example we consider here alloy scattering, for which [6]

$$
\frac{1}{\tau}=m^{*} \frac{\Omega_{0}}{\hbar^{3}}|\delta V|^{2} x(1-x) \int \psi^{4}(z) \mathrm{d} z,
$$

here $|\delta V|$ is the strength of the scattering potential, $\Omega_{0}$ is the volume of the unit cell and $x$ is the alloy composition parameter.

The relevant integral in the expression of the inverse relaxation time is now calculated as

$$
\int \psi_{n}^{4} \mathrm{~d} z=b_{n} \frac{n}{2^{5 / n}} \frac{\Gamma(5 / n)}{[\Gamma(3 / n)]^{2}} .
$$

For the Fang-Howard wave function (e.g. Eq. (4) with $n=1$ ) the well known result $[5,6]$ was recovered, i.e.

$$
\int \psi_{\mathrm{FH}}^{4} \mathrm{~d} z=\frac{3}{16} b=0.34071 \frac{e F}{\hbar^{2} / 2 m^{*}} .
$$

For the optimized trial function discussed above we obtained the numerical constant 0.41612 , a value significantly greater than that in Eq. (10). Not surprisingly, the use of the Takada-Uemura wave function also yields an enhanced value close to this, i.e. 0.39752 .

\section{Discussion and conclusions}

As it has been shown above, a simple (quasi-)one-parameter wave function could be constructed, which gave more accurate value for the ground subband energy in a triangular potential well, i.e. only 0.0044 per cent above the exact value, than the time honoured and widely used Fang-Howard wave function, or even the Takada-Uemura wave function.

The improvement on accuracy of the subband energy might not seem too significant in the light of the full self-consistent results (e.g. $[10,11])$. However, the use of a more correct wave function can have important consequences in the calculation of scattering rates and relaxation times, as demonstrated here for the case of short range potential scattering, an example of which is the alloy disorder scattering in ternary compounds. Taking the result obtained with the Fang-Howard 
wave function as a base-line, the inverse relaxation time, i.e. the scattering rate was found to be about 22 per cent and 16 per cent greater for the best optimized wave function and for the Takada-Uemura wave function respectively. This means that the standard formula based on the Fang-Howard wave function greatly underestimates the scattering rate and overestimates the mobility.

\section{References}

[1] L. Landau, E. Lifshitz, Quantum Mechanics, Addison-Wesley, New York 1958.

[2] F.F. Fang, W.E. Howard, Phys. Rev. Lett. 16, 797 (1966).

[3] F. Stern, W.E. Howard, Phys. Rev. 163, 816 (1967).

[4] F. Stern, Phys. Rev. B 5, 4891 (1972).

[5] T. Ando, A.B. Fowler, F. Stern, Rev. Mod. Phys. 54, 437 (1982).

[6] G. Bastard, Wave Mechanics Applied to Semiconductor Heterostructures, Les Editions de Physique, Les Ulis 1988.

[7] Y. Takada, Y. Uemura, J. Phys. Soc. Jap. 43, 139 (1977).

[8] F.J. Ohkawa, Y. Uemura, Surf. Sci. 58, 254 (1976).

[9] F.J. Ohkawa, Y. Uemura, J. Phys. Soc. Jap. 43, 917 (1977).

[10] T. Ando, J. Phys. Soc. Jap. 51, 3893 (1982).

[11] F. Stern, S. Das Sarma, Phys. Rev. B 30, 840 (1984). 